\title{
Inversions within restricted fillings of Young tableaux
}

\author{
Sarah Iveson* \\ Department of Mathematics \\ University of California, Berkeley, 970 Evans Hall \\ Berkeley, CA 94720-3840 \\ siveson@math. berkeley. edu
}

Submitted: Aug 1, 2005; Accepted: Jan 16, 2006; Published: Jan 25, 2006

Mathematics Subject Classifications: 05E10, 14M15

\begin{abstract}
In this paper we study inversions within restricted fillings of Young tableaux. These restricted fillings are of interest because they describe geometric properties of certain subvarieties, called Hessenberg varieties, of flag varieties. We give answers and partial answers to some conjectures posed by Tymoczko. In particular, we find the number of components of these varieties, give an upper bound on the dimensions of the varieties, and give an exact expression for the dimension in some special cases. The proofs given are all combinatorial.
\end{abstract}

\section{Introduction}

Our motivation is a result of Tymoczko in $[\mathrm{T}]$ which gives the Betti numbers of certain subvarieties of flag varieties in terms of the number of restricted fillings of a corresponding collection of tableaux. These Betti numbers specialize to objects of independent combinatorial interest, including Euler numbers and the rank of irreducible representations of the permutation group. The geometric properties which these restricted fillings describe have also been studied by de Mari-Shayman [DS] and by Fulman [F], and they also generalize classical combinatorial quantities, e.g., Euler numbers (see de Mari-ProcesiShayman [DPS]).

For the purposes of this paper, a Young tableau is a finite collection of $n$ boxes, arranged in rows of non-increasing length (from top to bottom), and a filling of a Young tableau is a labelling of the boxes in the tableau by the numbers from 1 to $n$ without repetition, subject to rules which will be described later. Our main interest will be to define and study a notion of combinatorial dimension for fillings of tableaux.

*This work was supported by the University of Michigan REU program. 
We also consider multitableaux $\lambda$, which are finite collections of tableaux arranged vertically. A Hessenberg function is a nondecreasing function $h:\{1,2, \ldots, n\} \rightarrow\{1,2, \ldots n\}$ satisfying the condition $h(\alpha) \geq \alpha$ for all $\alpha$.

Example 1.1. For $n=3$, the rule $h(1)=2, h(2)=2$, and $h(3)=3$ defines a Hessenberg function. But if we set $h(1)=1, h(2)=1$, and $h(3)=2$, then $h$ is not a Hessenberg function, because $2>h(2)$ and $3>h(3)$.

Given a Hessenberg function $h$, we will study fillings of multitableaux which are $h$ allowed (or simply allowed if the Hessenberg function $h$ is clear from the context). An $h$-allowed filling of a multitableau is a filling by the numbers $\{1, \ldots, n\}$ without repetition such that if \begin{tabular}{|l|l|l|}
\hline$\alpha$ & $\beta$
\end{tabular} appears in a tableau, then $\alpha \leq h(\beta)$.

Definition 1.2. The $h$-dimension (which we just call dimension, if $h$ is understood) of a filling of an $h$-allowed multitableau $\lambda$ is the sum of two quantities:

1. the number of pairs $(\alpha, \beta)$ in the filling of $\lambda$ such that

- $\alpha$ and $\beta$ are in the same tableau,

- the box filled with $\alpha$ is to the left of or directly below the box filled with $\beta$,

- $\beta<\alpha$, and

- if $\gamma$ fills the box immediately to the right of $\beta$ then $\alpha \leq h(\gamma)$.

2. the number of pairs $(\alpha, \beta)$ in the filling of $\lambda$ such that

- $\alpha$ and $\beta$ are in different tableaux,

- the tableau containing $\alpha$ is below the tableau containing $\beta$, and

- $\beta<\alpha \leq h(\beta)$.

In $[\mathrm{T}]$, these fillings are shown to be in bijective correspondence with cells of a decomposition of a Hessenberg variety, which is a subvariety of a flag variety, where the $h$-dimension of a filling is the dimension of the corresponding cell. As a special case, the Springer fibers, which arise naturally in geometric representation theory, occur when $h(\alpha)=\alpha$, and $\lambda$ consists of a single tableau.

Example 1.3. Suppose we have the following Hessenberg function

\begin{tabular}{c||c|c|c|c|c|c}
$\alpha$ & 1 & 2 & 3 & 4 & 5 & 6 \\
\hline$h(\alpha)$ & 2 & 4 & 4 & 5 & 5 & 6
\end{tabular}

and the following allowed filling

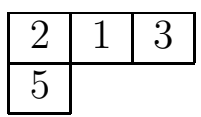

\begin{tabular}{|l|}
\hline 4 \\
\hline 6 \\
\hline
\end{tabular}

then using the above formula for the dimension, we see that the dimension is 5 , from the pairs $(2,1),(4,2),(4,3),(5,3)$, and $(6,4)$. 
The following definition, which relates to the Hessenberg function, will be needed in Sections 5 and 3 .

Definition 1.4. Each Hessenberg function $h:\{1, \ldots, n\} \rightarrow\{1, \ldots, n\}$ partitions the set $\{1, \ldots, n\}$ into blocks according to the rule that $\alpha+1$ is in the same block as $\alpha$ if and only if $h(\alpha)>\alpha$. We say that the Hessenberg function has $h$-blocks of sizes $n_{1}, n_{2}, \ldots, n_{k}$ if the $i^{\text {th }}$ block contains $n_{i}$ numbers.

In Section 3 we study the zero-dimensional fillings of a multitableau, the number of which is equal to the number of components of a corresponding Hessenberg variety. We describe all possible zero-dimensional fillings in terms of permutations of multisets which have a particular descent set.

Recall that the descent set of a permutation $\pi=\pi_{1} \pi_{2} \cdots \pi_{s}$ of (not necessarily distinct) positive integers $\pi_{1}, \pi_{2}, \ldots, \pi_{s}$ is the set $D(\pi)=\left\{i: \pi_{i}>\pi_{i+1}\right\}$, where the permutation is given using functional notation.

Theorem 1.5. If the Hessenberg function $h$ has $h$-blocks of sizes $n_{1}, n_{2}, \ldots, n_{k}$, then the number of zero-dimensional fillings of a multitableau $\lambda$ with $s_{i}$ boxes in the $i^{\text {th }}$ tableau is equal to the number of permutations $\pi$ of the multiset $\left\{1^{n_{1}}, 2^{n_{2}}, \ldots, k^{n_{k}}\right\}$ such that $D(\pi) \subseteq\left\{s_{1}, s_{1}+s_{2}, s_{1}+s_{2}+s_{3}, \ldots, s_{1}+s_{2}+\cdots+s_{\ell-1}\right\}$.

We also prove the following proposition, which demonstrates a kind of duality with an unexplained geometric interpretation. The significance of this duality is an open question.

Proposition 1.6. The number of zero-dimensional fillings of a multitableau with $s_{i}$ boxes in the $i^{\text {th }}$ tableau when the Hessenberg function has $h$-blocks of sizes $n_{1}, n_{2}, \ldots, n_{k}$ is the same as the number of zero-dimensional fillings of a multitableau with $n_{i}$ boxes in the $i^{\text {th }}$ tableau when the Hessenberg function has $h$-blocks of sizes $s_{1}, s_{2}, \ldots, s_{\ell}$.

In Section 2, we define certain properties of entries in a filling, and express the dimension in terms of entries with these properties. In Section 4, we use our expressions for the dimension in terms of these properties to prove the following theorem, which gives a sharp upper bound for the dimension of any filling. The upper bound we give consists of two summands, which arise in other combinatorial contexts.

Theorem 1.7. If the $j^{\text {th }}$ row of the $i^{\text {th }}$ tableau has length $d_{i, j}$, then the dimension of a filling is at most

$$
\sum_{\alpha=1}^{n}(h(\alpha)-\alpha)+\sum_{i=1}^{\ell} \sum_{j=2}^{k_{i}}(j-1) d_{i, j} .
$$

We then describe a special case when this upper bound is actually achieved in Section 5 , and we prove a sharper upper bound when the Hessenberg function is $h(\alpha)=\alpha+1$ in Section 6 . If $k_{\max }$ is the largest number of rows in any tableau of a multitableau $\lambda$, then we prove the following:

Theorem 1.8. When $h(\alpha)=\alpha+1$, the dimension of any filling is at most

$$
n-k_{\max }+\sum_{i=1}^{\ell} \sum_{j=2}^{k_{\ell}}(j-1) d_{i, j}
$$


and this upper bound is achieved.

In Section 7, we also study the dimension of another specific filling, which we call the big filling. This filling, when allowed, corresponds to the intersection of the big cell in the full flag variety with the Hessenberg variety. We find the dimension of the filling in this case and conjecture that it is in fact the filling of largest possible dimension, when allowed.

The author would like to thank Julianna Tymoczko for many helpful conversations and comments on earlier versions of this paper. Trevor Arnold and the referee, Bruce Sagan, also provided valuable feedback.

\section{Notation and conventions}

Throughout this paper, we will use the Greek letters $\alpha, \beta, \gamma, \delta, \epsilon$, and $\zeta$ to denote entries in a filling of a multitableau, and will use $\lambda$ to denote a multitableau. When we say that an entry $\alpha$ is to the left of an entry $\beta$ in a filling, this means that $\alpha$ and $\beta$ are in the same tableau, and the column containing $\alpha$ is to the left of the column containing $\beta$, and similarly for to the right of, above, and below. When we say that an entry $\alpha$ is directly above an entry $\beta$, this means that $\alpha$ and $\beta$ are in the same tableau, and $\alpha$ is somewhere above and in the same column as $\beta$, and similarly for directly below, directly to the left of, and directly to the right of. When we say an entry $\alpha$ is immediately to the right of an entry $\beta$, this means that $\alpha$ and $\beta$ are in the same tableau, $\alpha$ is in the same row as $\beta$, but in the box which is just to the right of the one containing $\beta$, and similarly for immediately to the left of, immediately below, and immediately above. Also, when we refer to the $i^{\text {th }}$ tableau in a multitableau $\lambda$, we count the tableaux from the top downward.

\section{An alternate expression for the dimension}

The following definitions will allow us to characterize the dimension of a filling in terms of entries which have certain properties which we call $P_{\alpha}$ and $D_{\alpha}$.

Definition 2.1. If an entry $\beta$ has the property that $\beta<\alpha \leq h(\beta)$ for some other entry $\alpha$, where $\alpha$ can be anywhere else in the filling, then we say that $\beta$ is $P_{\alpha}$.

Definition 2.2. We say that an entry $\beta$ is $D_{\alpha}$ if $\beta<\alpha$ for some other entry $\alpha$ which can occur anywhere else in the filling, and either $\beta$ fills the farthest right box of a row, or else if $\gamma$ is in the box immediately to the right of $\beta$, then $\alpha \leq h(\gamma)$.

In the remainder of this section, we describe properties of entries which are $P_{\alpha}$ and $D_{\alpha}$. We also express the dimension of a filling in terms of those entries which are $P_{\alpha}$ and $D_{\alpha}$, which will be useful throughout this paper.

Lemma 2.3 (Slide Right Lemma). Fix $\alpha \in\{1, \ldots, n\}$. If $\beta_{1}, \beta_{2}, \ldots, \beta_{k}$ appear consecutively in a row as in Figure 1 with $\beta_{k}<\alpha \leq h\left(\beta_{1}\right)$, then at least one of $\beta_{1}, \beta_{2}, \ldots, \beta_{k}$ is $P_{\alpha}$. 


\begin{tabular}{|l|l|l|l|l|l|l|}
\hline$\cdots$ & $\beta_{1}$ & $\beta_{2}$ & $\cdots$ & $\beta_{k-1}$ & $\beta_{k}$ & $\cdots$ \\
\hline
\end{tabular}

Figure 1: Entries appearing consecutively in a row.

Proof. We know that $\alpha \leq h\left(\beta_{1}\right)$ so if $\alpha>\beta_{1}$ then $\beta_{1}$ is $P_{\alpha}$. Otherwise $\alpha \leq \beta_{1} \leq h\left(\beta_{2}\right)$ since all tableaux we are considering have \begin{tabular}{|l|l|l|}
\hline$\beta_{1}$ & $\beta_{2}$ \\
\hline
\end{tabular} only if $\beta_{1} \leq h\left(\beta_{2}\right)$, so slide to the right and decide if $\beta_{2}$ is $P_{\alpha}$. Continuing this "sliding right" procedure, either one of $\beta_{1}, \beta_{2}, \ldots, \beta_{k-1}$ is $P_{\alpha}$ or else $\alpha \leq \beta_{k-1} \leq h\left(\beta_{k}\right)$. In the latter case, the assumption that $\alpha>\beta_{k}$ implies that $\beta_{k}$ is $P_{\alpha}$.

Corollary 2.4. Fix $\alpha$. If $\beta_{0}, \beta_{1}, \beta_{2}, \ldots, \beta_{k}$ appear in a row, with $\alpha \neq \beta_{0}$ and $\alpha \neq \beta_{k}$, where $\beta_{0}$ and $\beta_{k}$ are $D_{\alpha}$, then one of $\beta_{1}, \beta_{2}, \ldots, \beta_{k}$ is $P_{\alpha}$.

Proof. Since $\beta_{0}$ and $\beta_{k}$ are $D_{\alpha}$, we know that $\beta_{k}<\alpha \leq h\left(\beta_{1}\right)$. Our claim then follows from the Slide Right Lemma.

Lemma 2.5 (Slide Left Lemma). Fix $\alpha \in\{1, \ldots, n\}$. If $\beta_{1}, \beta_{2}, \ldots, \beta_{k-1}$ are arranged consecutively in a row as in Figure $1, \alpha>\beta_{1}$, and either

- $\beta_{k-1}$ is at the end of the row or

- the box directly to the right of $\beta_{k-1}$ is filled with a number $\beta_{k}$ such that $\alpha \leq h\left(\beta_{k}\right)$, then at least one of $\beta_{1}, \beta_{2}, \ldots, \beta_{k-1}$ is $D_{\alpha}$.

Proof. If $\alpha>\beta_{k-1}$, then as either $\beta_{k-1}$ is at the end of a row or else $\alpha \leq h\left(\beta_{k}\right)$, we see that $\beta_{k-1}$ is $D_{\alpha}$. Otherwise $\alpha \leq \beta_{k-1} \leq h\left(\beta_{k-1}\right)$ so slide to the left and decide if $\beta_{k-2}$ is $D_{\alpha}$. Continuing this "sliding left" procedure, either one of $\beta_{2}, \ldots, \beta_{k-1}$ is $D_{\alpha}$ or else $\alpha \leq \beta_{2} \leq h\left(\beta_{2}\right)$. In the latter case, the assumption that $\alpha>\beta_{1}$ implies that $\beta_{1}$ is $D_{\alpha}$.

Corollary 2.6. Fix $\alpha$. If $\beta_{1}, \beta_{2}, \ldots, \beta_{k-1}$ appear consecutively in a row where $\beta_{1}$ is $P_{\alpha}$ and either $\beta_{k-1}$ is at the end of the row or $\beta_{k}$ is directly to the right of $\beta_{k-1}$ and $\beta_{k}$ is $P_{\alpha}$, then one of $\beta_{1}, \beta_{2}, \ldots, \beta_{k-1}$ is $D_{\alpha}$.

Proof. Since $\beta_{1}$ is $P_{\alpha}$ we have $\alpha>\beta_{1}$, so the claim follows from the Slide Left Lemma.

By Corollaries 2.4 and 2.6, the entries which are $D_{\alpha}$ and $P_{\alpha}$ alternate. For example, if two entries are $P_{\alpha}$ in the same row, as in Figure 2, there will be an entry which is $D_{\alpha}$ in the specified region. An analogous property for $D_{\alpha}$ entries also holds (see Figure 2). Hence if there are two $P_{\alpha}$ entries with no other $P_{\alpha}$ entries between them, there is exactly one $D_{\alpha}$ entry in the specified region, and vice versa.

Remark 2.7. Note the following:

- After the last $P_{\alpha}$ entry, there is exactly one $D_{\alpha}$ entry, which is at the $P_{\alpha}$ entry or strictly to the right of it, by the Slide Left Lemma or Corollary 2.6. 


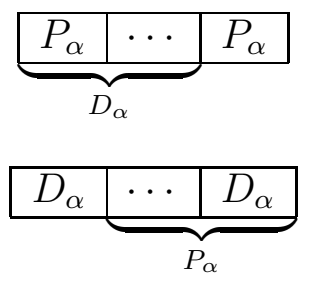

Figure 2: $P_{\alpha}$ and $D_{\alpha}$ entries

- Before the first $P_{\alpha}$ entry, there are either zero or one $D_{\alpha}$ entries by Corollary 2.4.

- Consider the row in which the entry $\alpha$ occurs. By the Slide Right Lemma, there are no $D_{\alpha}$ entries to the right of $\alpha$ and before the first $P_{\alpha}$ entry which is to the right of $\alpha$. Indeed, if $\beta_{1}$ is immediately to the right of $\alpha$, we have $\alpha \leq h\left(\beta_{1}\right)$, so suppose there is some entry $\beta_{k}$ which is $D_{\alpha}$ and before the first $P_{\alpha}$ entry to the right of $\alpha$. Then by applying the Slide Right Lemma, we find that there is some entry which is $P_{\alpha}$ to the right of $\alpha$ which is before the first $P_{\alpha}$ entry, a contradiction.

Definition 2.8. We will say that an entry $\beta$ which is $D_{\alpha}$ has no contributing $P_{\alpha}$ if the following conditions hold:

- $\alpha$ is directly below or to the left of $\beta$, and

- if $\beta_{0}$ is the entry which is in the same row as $\beta$ and in the same column as $\alpha$ (see Figure 3), then any entry which is between $\beta_{0}$ and $\beta$, inclusive, is not $P_{\alpha}$.

Note that if $\alpha$ is directly below $\beta$, then $\beta=\beta_{0}$. Also, $\alpha$ could in fact be directly above $\beta_{0}$; Figure 3 only shows the case where $\alpha$ is below $\beta$, but $\alpha$ could in fact be above $\beta$.

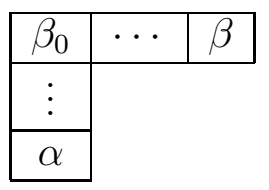

Figure 3: For $\alpha$ below and to the left of $\beta$. $\beta$ is $D_{\alpha}$ with no contributing $P_{\alpha}$ if $\beta_{0}$ and $\beta$ are not $P_{\alpha}$, and there is no $P_{\alpha}$ entry between $\beta_{0}$ and $\beta$.

We now express the dimension of a filling in terms of entries which are $P_{\alpha}$ and $D_{\alpha}$ in two different ways.

Corollary 2.9. The dimension of a filling is the sum of the two quantities:

1. the number of pairs $(\alpha, \beta)$ in the filling of $\lambda$ such that 
- $\alpha$ and $\beta$ are in the same tableau,

- $\alpha$ is to the left of or directly below the box filled with $\beta$, and

- $\beta$ is $D_{\alpha}$;

2. the number of pairs $(\alpha, \beta)$ in $\lambda$ such that

- $\alpha$ and $\beta$ are in different tableaux,

- the tableau containing $\alpha$ is below the tableau containing $\beta$, and

- $\beta$ is $P_{\alpha}$.

Corollary 2.10. The dimension of a filling is equal to the sum of the three quantities:

1. the number of pairs $(\alpha, \beta)$ in the filling of $\lambda$ such that

- $\alpha$ and $\beta$ are in the same tableau,

- the box filled with $\alpha$ is to the left of or directly below the box filled with $\beta$,

- $\beta$ is $P_{\alpha}$.

2. the number of pairs $(\alpha, \beta)$ in the filling of $\lambda$ such that

- $\alpha$ and $\beta$ are in the same tableau,

- the box filled with $\alpha$ is to the left of or directly below the box filled with $\beta$,

- $\beta$ is $D_{\alpha}$ with no contributing $P_{\alpha}$.

3. the number of pairs $(\alpha, \beta)$ in $\lambda$ such that

- $\alpha$ and $\beta$ are in different tableaux,

- the tableau containing $\alpha$ is below the tableau containing $\beta$, and

- $\beta$ is $P_{\alpha}$.

Proof. Using the formula for the dimension given in Definition 1.2, the last two conditions of the first part are equivalent to $\beta$ being $D_{\alpha}$. Also by definition, the second quantity is exactly the second quantity in Corollary 2.9. Thus we get the expression for the dimension of a filling given in Corollary 2.9 .

Since $P_{\alpha}$ and $D_{\alpha}$ entries alternate the first quantity in Corollary 2.9 is equivalent to the number of pairs $(\alpha, \beta)$ in the filling of $\lambda$ such that

- $\alpha$ and $\beta$ are in the same tableau,

- the box filled with $\alpha$ is to the left of or directly below the box filled with $\beta$, and

- either $\beta$ is $D_{\alpha}$ with no contributing $P_{\alpha}$, or else $\beta$ is $P_{\alpha}$.

From this, we get the expression for the dimension given in Corollary 2.10. 


\section{$3 \quad$ Zero-Dimensional Fillings}

The number of zero-dimensional fillings is of interest because it is the number of connected components of a corresponding Hessenberg variety. In this section, we prove that there is a bijection between zero-dimensional fillings and permutations of a multiset with a fixed descent set. In this section we also prove some facts about how the number of zero-dimensional fillings relates to the sizes of $h$-blocks (Definition 1.4), and give closed formulas for the number of zero-dimensional fillings in special cases.

If a multitableau $\lambda$ has $\ell$ tableaux with $s_{i}$ boxes in the $i^{\text {th }}$ tableau, then we define the base filling to have the first $s_{\ell}$ numbers in the bottom tableau, the next $s_{\ell-1}$ numbers in the next tableau up, the next $s_{\ell-2}$ numbers in the next tableau, and so on. Each tableau is then filled according to the rule that the smallest number is in the bottom entry of the left column, and if $\alpha$ fills a box not in the top row of a tableau, then $\alpha+1$ is directly above it. If $\alpha$ is in the top row of a tableau, then $\alpha+1$ is in the bottom entry of the column to the right of the one containing $\alpha$. An example of this filling is seen in Figure 4 .

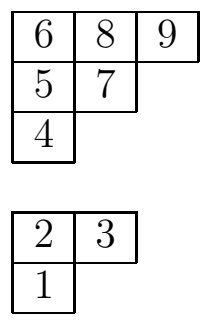

Figure 4: An example of the base filling.

Definition 3.1. If every tableau of $\lambda$ is filled according to the following rule, we call the filling a pseudo-base filling. Suppose the $i^{\text {th }}$ tableau of $\lambda$ has $k_{i}$ boxes. If $\left\{\alpha_{1}^{i}, \ldots, \alpha_{k}^{i}\right\}$ are the numbers appearing in the filling of the $i^{\text {th }}$ tableau and $\alpha_{1}^{i}<\alpha_{2}^{i}<\cdots<\alpha_{k}^{i}$, then this tableau is filled as follows: $\alpha_{1}^{i}$ is in the bottom of the left column, and if some $\alpha_{r}^{i}$ is in an entry not in the top row, $\alpha_{r+1}^{i}$ is directly above it. If $\alpha_{r}^{i}$ is in the top row, then $\alpha_{r+1}^{i}$ is in the bottom entry of the column to the right of the one containing $\alpha_{r}^{i}$.

In general, a pseudo-base filling on a multitableau $\lambda$ depends on which entries are in each tableau, so it is not unique. However, given a set of numbers for each tableau, there is a unique pseudo-base filling.

Proposition 3.2. If a filling of $\lambda$ is zero-dimensional, then it must be a pseudo-base filling.

Proof. Suppose that some zero-dimensional filling is not a pseudo-base filling. Then for some $i$, the $i^{\text {th }}$ tableau is not filled in this way. Then there will be some pair $\left(\alpha, \beta_{1}\right)$ such that $\alpha>\beta_{1}$, and $\alpha$ is to the left of or directly below $\beta_{1}$. Let $\beta_{2}, \beta_{3}, \ldots, \beta_{k}$ be the numbers to the right of $\beta_{1}$, as seen below, with $\beta_{k}$ and the end of the row. 


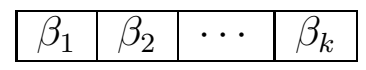

The box filled with $\alpha$ is to the left of or directly below all of these entries. By the Slide Left Lemma, one of $\beta_{1}, \beta_{2}, \ldots, \beta_{k}$ is $D_{\alpha}$, and the box filled with $\alpha$ is to the left of or directly below whichever entry is $D_{\alpha}$, so this pair will contribute to the dimension. Hence the dimension must be at least 1 .

Corollary 3.3. When $\lambda$ consists of only one tableau, there is only one zero-dimensional filling, the base filling.

Given any pseudo-base filling of a multitableau $\lambda$, any pair $(\alpha, \beta)$ with $\alpha$ and $\beta$ in the same tableau where $\alpha$ is in a box to the left of or directly below the box filled with $\beta$ has $\beta>\alpha$ by definition. Thus, any pair $(\alpha, \beta)$ that contributes to the dimension must have $\alpha$ and $\beta$ in different tableaux. It follows that the dimension of a pseudo-base filling is the number of pairs $(\alpha, \beta)$ such that $\alpha$ and $\beta$ are in different tableaux, the box filled with $\alpha$ is below the box filled with $\beta$, and $\beta<\alpha \leq h(\beta)$.

We write a permutation of the multiset $\left\{1^{n_{1}}, 2^{n_{2}}, \ldots, k^{n_{k}}\right\}$ as $\pi=\pi_{1} \pi_{2} \cdots \pi_{s}$ (using functional notation) where $s=n_{1}+n_{2}+\cdots+n_{k}$ and each $\pi_{i} \in\left\{1^{n_{1}}, 2^{n_{2}}, \ldots, k^{n_{k}}\right\}$. The descent set of such a permutation $\pi$ is $D(\pi)=\left\{i: \pi_{i}>\pi_{i+1}\right\}$.

Theorem 3.4. For a Hessenberg function $h$ with $h$-blocks of sizes $n_{1}, n_{2}, \ldots, n_{k}$ and a multitableau $\lambda$ with tableaux of sizes $s_{1}, s_{2}, \ldots, s_{\ell}$, the number of zero-dimensional fillings of $\lambda$ is equal to the number of permutations $\pi$ of the multiset $\left\{1^{n_{1}}, 2^{n_{2}}, \ldots, k^{n_{k}}\right\}$ whose descent set $D(\pi)$ is contained in $\left\{s_{1}, s_{1}+s_{2}, s_{1}+s_{2}+s_{3}, \ldots, s_{1}+s_{2}+\cdots+s_{\ell-1}\right\}$.

Proof. We will prove this by constructing a bijection which sends a permutation $\pi$ of the multiset $\left\{1^{n_{1}}, 2^{n_{2}}, \ldots, k^{n_{k}}\right\}$ whose descent set $D(\pi)$ is contained in $\left\{s_{1}, s_{1}+s_{2}, s_{1}+\right.$ $\left.s_{2}+s_{3}, \ldots, s_{1}+s_{2}+\cdots+s_{\ell-1}\right\}$ to a zero-dimensional filling. Write $\pi=\pi_{1} \pi_{2} \cdots \pi_{s}$ where $s=s_{1}+s_{2}+\cdots+s_{\ell}$. Look at the first $s_{1}$ numbers $\pi_{1}, \pi_{2}, \ldots, \pi_{s_{1}}$. Let $c_{1,1}$ be the number of $1 \mathrm{~s}, c_{1,2}$ be the number of $2 \mathrm{~s}, \ldots, c_{1, k}$ be the number of $k \mathrm{~s}$. For each $i$, place the largest $c_{1, i}$ numbers of the $i$ th $h$-block in the top tableau, with the pseudo-base filling for these numbers, which will fill the top tableau. Then for each $i$, place the largest unused $c_{2, i}$ numbers of the $i^{\text {th }} h$-block in the second tableau, with the pseudo-base filling on these numbers as well. Repeat this process, which fills each tableau completely, since $c_{j, 1}+c_{j, 2}+\cdots+c_{j, k}=s_{j}$ for each $j$. Continuing this process will fill the multitableau with $1, \ldots, n$ since there were $n_{j} j \mathrm{~s}$ in the multiset. If $\alpha$ and $\beta$ are in the same $h$-block and $\alpha>\beta$, the filling places $\alpha$ either in the same tableau as or in a tableau above $\beta$. Moreover, each tableau is filled with a pseudo-base filling, so this filling of $\lambda$ is guaranteed to be an allowed zero-dimensional filling.

Suppose given two permutations of the multiset $\pi$ and $\pi^{\prime}$ whose descent sets are contained in $\left\{s_{1}, s_{1}+s_{2}, \ldots, s_{1}+s_{2}+\cdots+s_{\ell-1}\right\}$. If $\pi \neq \pi^{\prime}$, then the first $s_{1}$ numbers of $\pi$, or the $s_{2}$ after those, or the next $s_{3}$, or so on, will have a different number of $i$ s than the same group of $s_{j}$ numbers in $\pi^{\prime}$, for some $i$. Thus, the filling that $\pi$ is sent to has a different number of entries from the $i^{\text {th }} h$-block in the $j^{\text {th }}$ tableau than the filling corresponding to $\pi^{\prime}$. This means the map is injective. 
The map is also surjective: in order for an allowed filling with $c_{j, i}$ numbers from the $i^{\text {th }} h$-block in the $j^{\text {th }}$ tableau be zero-dimensional, it must be that whenever $\alpha$ and $\alpha+1$ are in the same $h$-block, $\alpha$ is either in the same tableau as $\alpha+1$ or in a tableau below it. So if we let $\pi$ be the permutation which has $c_{1,1} 1 \mathrm{~s}, c_{1,2} 2 \mathrm{~s}, \ldots, c_{1, k} k \mathrm{~s}$ in the first group of $s_{1}$ numbers, $c_{2,1} 1$ 's, $c_{2,2} 2$ 's, $\ldots, c_{2, k}$ k's in the next group of $s_{2}$ numbers, and so on, then $\pi$ will correspond to this filling.

Example 3.5. Consider the filling seen in Figure 5 and suppose the $h$-blocks are $\{1,2,3,4\}$, $\{5\}$, and $\{6,7\}$; since the top tableau contains two entries from the first $h$-block, one entry from the second $h$-block, and one entry from the third $h$-block, the corresponding permutation $\pi$ must start with 1123. The next tableau down has two entries from the first $h$-block and one entry from the third $h$-block, so the next three numbers of $\pi$ are 113. Thus $\pi=1123113$.

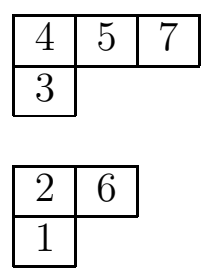

Figure 5: The filling associated to $\pi=1123113$, with $h$-blocks $\{1,2,3,4\},\{5\}$, and $\{6,7\}$.

Recall from [S, Section 1.3] that the total number of permutations of the multiset $\left\{1^{n_{1}}, 2^{n_{2}}, \ldots, k^{n_{k}}\right\}$ where $n=n_{1}+n_{2}+\cdots+n_{k}$ is $\left(\begin{array}{c}n \\ n_{1}, n_{2}, \ldots, n_{k}\end{array}\right)$. The following Corollary gives special cases of the previous theorem.

Corollary 3.6. We can easily describe the number of zero-dimensional fillings in the following cases:

1. If $s_{1}=n$, there is only one tableau, so the base filling is the only zero-dimensional filling.

2. If $n_{1}=n$, the Hessenberg function satisfies $h(\alpha)>\alpha$ for all $\alpha$, so there is only one $h$-block, and the base filling is the only zero-dimensional filling.

3. If $s_{i}=1$ for all $i$, the multitableau consists of $n$ tableaux, each containing only one box, so there are $\left(\begin{array}{c}n \\ n_{1}, n_{2}, \ldots, n_{k}\end{array}\right)$ zero-dimensional fillings.

4. If $n_{i}=1$ for all $i, h(\alpha)=\alpha$ for all $\alpha$, so there are $\left(\begin{array}{c}n \\ s_{1}, s_{2}, \ldots, s_{\ell}\end{array}\right)$ zero-dimensional fillings.

5. If $n_{i}=1$ for all $i$ and $s_{j}=1$ for all $j$, the multitableau consists of $n$ tableaux, each a single box, and $h(\alpha)=\alpha$ for all $\alpha$, so there are $n$ ! zero-dimensional fillings.

The following Proposition establishes a duality between 0-dimensional fillings; a geometric interpretation for this duality is unknown. 
Proposition 3.7. The number of zero-dimensional fillings corresponding to a Hessenberg function with $h$-blocks of sizes $n_{1}, \ldots, n_{k}$ and multitableau $\lambda$ with tableaux of sizes $s_{1}, \ldots, s_{\ell}$ is equal to the number of zero-dimensional fillings corresponding to a Hessenberg function with $h$-blocks of sizes $s_{1}, \ldots, s_{\ell}$ and multitableau $\lambda^{\prime}$ with tableaux of sizes $n_{1}, \ldots, n_{k}$.

Proof. We will prove this by constructing a bijection between the permutations $\pi$ of the multiset $\left\{1^{n_{1}}, 2^{n_{2}}, \ldots, k^{n_{k}}\right\}$ such that $D(\pi) \subseteq\left\{s_{1}, s_{1}+s_{2}, \ldots, s_{1}+s_{2}+\cdots+s_{\ell-1}\right\}$, and permutations $\sigma$ of the multiset $\left\{1^{s_{1}}, 2^{s_{2}}, \ldots, l^{s_{\ell}}\right\}$ such that $D(\sigma) \subseteq\left\{n_{1}, n_{1}+n_{2}, \ldots, n_{1}+\right.$ $\left.n_{2}+\cdots+n_{k-1}\right\}$. Given $\pi$, group the numbers so that the first group contains the first $s_{1}$ numbers of $\pi$, the second contains the next $s_{2}$ numbers, the third contains the next $s_{3}$, and so on. If the $j^{\text {th }}$ group contains $c_{i, j}$ copies of $i$ (for $j=1, \ldots, \ell$ and $i=1, \ldots, k$ ) then put $c_{i, j}$ copies of $j$ in the $i^{\text {th }}$ group of $\sigma$, and then rearrange each group of $\sigma$ so that the numbers in the group are increasing. The first group of $\sigma$ contains the first $n_{1}$ numbers, the second contains the next $n_{2}$ numbers, and so on. This regrouping ensures that $D(\sigma) \subseteq\left\{n_{1}, n_{1}+n_{2}, \ldots, n_{1}+n_{2}+\cdots+n_{k-1}\right\}$, and since the $j^{\text {th }}$ group of $\pi$ contained $s_{j}$ numbers for each $j$, the permutation $\sigma$ will have $s_{j}$ copies of $j$ in it. This means it is in fact a permutation of $\left\{1^{s_{1}}, 2^{s_{2}}, \ldots, l^{s_{\ell}}\right\}$. This construction is well-defined and is symmetric in $\sigma$ and $\pi$ so it is in fact bijective.

Example 3.8. As example of this bijection, suppose $n_{1}=5, n_{2}=3, n_{3}=2$ and $s_{1}=3$, $s_{2}=3, s_{3}=2, s_{4}=2$. If $\pi=1121331122$, then there are two 1s in the first group, one 1 in the second group, and two 1s in the third group, so the first group of $\sigma$ is 11233 . We end up with $\sigma=1123314422$.

\section{An Upper Bound for the Dimension}

We use the description of the dimension in Corollary 2.10 to find a sharp upper bound for the dimension of a filling of $\lambda$. This upper bound is achieved in a special case which is described in Section 5. The expression for the upper bound comprises two terms. The first, $\sum_{\alpha=1}^{n}(h(\alpha)-\alpha)$, is a parameter associated to ad-nilpotent ideals and to regular nilpotent Hessenberg varieties [ST]. The second, $\sum_{i=1}^{\ell} \sum_{j=2}^{k_{i}}(j-1) d_{i, j}$ where $k_{i}$ is the number of entries in the $i^{\text {th }}$ tableau, and $d_{i, j}$ is the number of entries in the $j^{\text {th }}$ row of the $i^{\text {th }}$ tableau, is the maximal dimension when $h(\alpha)=\alpha$, which follows from Proposition 5.2.

In this section and the following, we use the following lemma, which expresses the sum $\sum_{j=2}^{k_{i}}(j-1) d_{i, j}$ in a slightly different way.

Lemma 4.1. If $C_{i}$ is the set of all columns in the $i^{\text {th }}$ tableau, and $\ell(c)$ is the length of column $c$, then for all $i$,

$$
\sum_{j=2}^{k_{i}}(j-1) d_{i, j}=\sum_{c \in C_{i}}\left(\begin{array}{c}
\ell(c) \\
2
\end{array}\right) .
$$

Proof. Let $k_{i}$ be the number of rows in the $i^{\text {th }}$ tableau, and $d_{i, j}$ be the number of entries in the $j^{\text {th }}$ row of the $i^{\text {th }}$ tableau. Then $\sum_{j=2}^{k_{i}}(j-1) d_{i, j}$ is the sum of the number of rows 
in the $i^{\text {th }}$ tableau which are above the box filled with $\gamma$ for each entry $\gamma$ in the $i^{\text {th }}$ tableau of $\lambda$. This sum can also be expressed as $\sum_{c \in C_{i}} 1+2+\cdots+(\ell(c)-1)=\sum_{c \in C_{i}}\left(\begin{array}{c}\ell(c) \\ 2\end{array}\right)$.

Theorem 4.2. Let $\lambda$ be a multitableau with $\ell$ tableaux, such that the $i^{\text {th }}$ tableau has $k_{i}$ rows and the length of the $j^{\text {th }}$ row of the $i^{\text {th }}$ tableau is $d_{i, j}$. Then the dimension of any filling of $\lambda$ is at most

$$
\sum_{\alpha=1}^{n}(h(\alpha)-\alpha)+\sum_{i=1}^{\ell} \sum_{j=2}^{k_{i}}(j-1) d_{i, j} .
$$

Proof. Let $(\alpha, \beta)$ be a pair contributing to the dimension as in Corollary 2.10. We want to compute an upper bound on the number of such pairs, which gives us an upper bound for the dimension of any filling. First, note that if $\beta$ is $P_{\alpha}$, then $\beta<\alpha \leq h(\beta)$, so $\alpha \in\{\beta+1, \beta+2, \ldots, h(\beta)\}$, a total of $h(\beta)-\beta$ possibilities. Thus there are at most $\sum_{\beta=1}^{n} h(\beta)-\beta$ pairs $(\alpha, \beta)$ such that $\beta$ is $P_{\alpha}$. Also note that for any pair $(\alpha, \beta)$ where $\beta$ is $P_{\alpha}$, this pair can contribute to either part (1) or part (3) of the expression for the dimension in Corollary 2.10, but not both. So parts (1) and (3) can contribute at most $\sum_{\beta=1}^{n} h(\beta)-\beta$ to the dimension.

We show that the number of pairs satisfying the condition in part (2) is at most $\sum_{i=1}^{\ell} \sum_{j=2}^{k_{i}}(j-1) d_{i, j}$, so the sum of parts $(1),(2)$, and (3) is at most $\sum_{\alpha=1}^{n}(h(\alpha)-\alpha)+$ $\sum_{i=1}^{\ell} \sum_{j=2}^{k_{i}}(j-1) d_{i, j}$.

Given a fixed $\alpha$, there is at most one $D_{\alpha}$ entry with no contributing $P_{\alpha}$. Given any two entries $\alpha_{1}$ and $\beta_{1}$ which are both in the same column, as in Figure 6, we now show that at most one of the following can occur:

- there is an entry $\beta_{k}$ in the same row as $\beta_{1}$ which is $D_{\alpha_{1}}$, the pair $\alpha_{1}, \beta_{k}$ contributes to the dimension, and $\beta_{k}$ has no contributing $P_{\alpha_{1}}$ or

- there is an entry $\alpha_{\ell}$ in the same row as $\alpha_{1}$ which is $D_{\beta_{1}}$, the pair $\beta_{1}, \alpha_{\ell}$ contributes to the dimension, and $\alpha_{\ell}$ has no contributing $P_{\beta_{1}}$.

Indeed suppose there is both a $\beta_{k}$ and an $\alpha_{\ell}$ satisfying the given conditions. In order for $\alpha_{1}, \beta_{k}$ to contribute to the dimension, $\alpha_{1}$ is to the left of or directly below $\beta_{k}$, so $k \geq 1$. For $\beta_{1}, \alpha_{\ell}$ to contribute to the dimension, $\beta_{1}$ must be to the left of $\alpha_{\ell}$ (since $\alpha_{1}$ is below $\left.\beta_{1}\right)$, so $\ell>1$.

\begin{tabular}{|c|c|c|}
\hline$\beta_{1}$ & $\cdots$ & $\beta_{k}$ \\
\hline$\vdots$ & \multicolumn{2}{|c}{} \\
\hline$\alpha_{1}$ & $\cdots$ & $\alpha_{\ell}$ \\
\hline
\end{tabular}

Figure 6: Note that this shows the case where $\ell<k$, but it could also be that $\ell \geq k$.

Suppose that $\beta_{1}<\alpha_{1}$. Then $\beta_{1}<\alpha_{1} \leq h\left(\alpha_{2}\right)$, and $\beta_{1}>\alpha_{\ell}$ since $\alpha_{\ell}$ is $D_{\beta_{1}}$. By the Slide Right Lemma, one of $\alpha_{2}, \ldots, \alpha_{\ell}$ is $P_{\beta_{1}}$ and thus $\alpha_{\ell}$ has a contributing $P_{\alpha_{1}}$. Otherwise, 
if $\alpha_{1}<\beta_{1}$, we have $\alpha_{1}<\beta_{1} \leq h\left(\beta_{1}\right)$, and $\alpha_{1}>\beta_{k}$ since $\beta_{k}$ is $D_{\alpha_{1}}$. Again by the Slide Right Lemma, one of $\beta_{1}, \ldots, \beta_{k}$ is $P_{\alpha_{1}}$ and thus $\beta_{k}$ has a contributing $P_{\beta_{1}}$.

If two entries $\alpha$ and $\beta$ are in the same column, then it cannot happen that both there is an entry in the same row as $\alpha$ which is $D_{\beta}$ with no contributing $P_{\beta}$ and there is an entry in the same row as $\beta$ which is $D_{\alpha}$ with no contributing $P_{\alpha}$. Since there are $\sum_{i=1}^{\ell} \sum_{j=2}^{k_{i}}(j-1) d_{i, j}$ pairs of such entries $\alpha$ and $\beta$, by Lemma 4.1 , this is also the maximum number of pairs satisfying the condition in part (2) of Corollary 2.10.

\section{The dimension in a special case}

In this section we demonstrate that the upper bound in Theorem 4.2 can be achieved.

Definition 5.1. Let $s_{i}$ be the number of boxes in the $i^{\text {th }}$ tableau, $k_{i}$ the number of rows in the $i^{\text {th }}$ tableau, $d_{i, j}$ the number of entries in the $j^{\text {th }}$ row of the $i^{\text {th }}$ tableau, and let $\ell$ be the number of tableaux in $\lambda$. We say that $h$ is nicely partitioned with respect to $\lambda$ if it is the case that for $0 \leq m \leq \ell$ and $1 \leq t \leq k_{m}$ that

$$
h\left(\sum_{i=1}^{m-1} s_{i}+\sum_{j=1}^{t} d_{m, j}\right)=\sum_{i=1}^{m-1} s_{i}+\sum_{j=1}^{t} d_{m, j} .
$$

The property of $h$ being nicely partitioned with respect to $\lambda$ means that the Hessenberg function $h$ has the property that if the first $d_{1,1}$ numbers are put in the first row, and the next $d_{1,2}$ numbers are put in the second row, and so on, the $h$-blocks are not broken up between rows. Note that the Hessenberg function is not determined by this condition. One example that always satisfies this condition is $h(\alpha)=\alpha$.

For example if $d_{1,1}=4, d_{1,2}=2, d_{2,1}=2$, and $d_{2,2}=1$, then for $h$ to be nicely partitioned we must have $h(4)=4, h(6)=6, h(8)=8$, and $h(9)=9$. The multitableau for this example can be seen in Figure 7.

Consider the following filling: first, take the numbers $1, \ldots, n_{1}$ from the first $h$-block, and put them at the beginning of the first row in decreasing order. Then put the next $h$-block in decreasing order after the previous one, continuing until the first row is filled. Each row that is filled in this way will end exactly after some $h$-block is used. After the first row is full, go to the second row and put the next $h$-block in decreasing order, then the next $h$-block in decreasing order after it, skipping to the top row of the next tableau after each one is filled, and continue this process until every row of every tableau is filled. We will call this the $h$-block filling. Note that with this filling $h$-blocks are not broken up between rows.

An example of this filling can be seen in Figure refhblock) in the case when is when $d_{1,1}=4, d_{1,2}=2, d_{2,1}=2, d_{2,2}=1$, and the Hessenberg function is given by:

$$
\begin{array}{llrl}
h(1)=2 & h(2)=2 & h(3)=4 & \\
h(5)=5 & h(6)=6 & \\
h(7)=8 & h(8)=8 & \\
h(9)=9 & &
\end{array}
$$



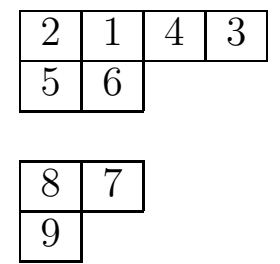

Figure 7: Example of the $h$-block filling.

Proposition 5.2. If $h$ is nicely partitioned with respect to $\lambda$, the dimension of the $h$-block filling is exactly

$$
\sum_{\delta=1}^{n}(h(\delta)-\delta)+\sum_{i=1}^{\ell} \sum_{j=2}^{k_{i}}(j-1) d_{i, j}
$$

Proof. It suffices to show that there are at least $\sum_{i=1}^{\ell} \sum_{j=2}^{k_{i}}(j-1) d_{i, j}+\sum_{\delta=1}^{n}(h(\delta)-\delta)$ pairs $(\alpha, \beta)$ which contribute to the dimension.

For each tableau and each entry $\alpha$ in a row which is not the top row, we have $\alpha>\beta$ whenever $\beta$ is at the end of a row which is above $\alpha$ and in the same tableau. Thus, the pair $(\alpha, \beta)$ contributes to the dimension. Given $\alpha$, there is one of these $\beta$ for each row above the row containing $\alpha$. So for each tableau $T$, there are $\sum_{\alpha \in T} r(\alpha)$ such pairs, where $r(\alpha)$ is the number of rows in $T$ above the row containing alpha, which, by the proof of Lemma 4.1 , shows that there are $\sum_{j=2}^{k_{i}}(j-1) d_{i, j}$ such pairs in the $i^{\text {th }}$ tableau. Summing over all tableaux, the number of pairs of this type contributing to the dimension is at least $\sum_{i=1}^{\ell} \sum_{j=2}^{k_{i}}(j-1) d_{i, j}$.

For each entry $\alpha$, count the number of entries $\beta$ to the right of $\alpha$ in the same row where $\beta$ is $P_{\alpha}$. If $\beta$ is $P_{\alpha}$ then $\beta<\alpha \leq h(\beta)$ and for each $\beta$, there are $h(\beta)-\beta$ possible entries $\alpha$ for which $\beta$ is $P_{\alpha}$. For all $\gamma>\beta$, we have $h(\gamma) \geq h(\beta)$. Since $\beta$ is $P_{\alpha}$, we know $h(\gamma) \geq h(\beta) \geq \alpha$. Therefore $h(\gamma)>\gamma$ for all $\gamma$ satisfying $\beta<\gamma<\alpha$. So for any such $\gamma$, both $\gamma$ and $\gamma+1$ are in the same $h$-block. Also since $h(\beta)>\beta$, we see that $\beta$ and $\beta+1$ are in the same $h$-block. It follows that $\beta, \beta+1, \ldots, \alpha$ are all in the same $h$-block. Since all of these are to the left of $\beta$ by the hypothesis, there are at least $\sum_{\delta=1}^{n}(h(\delta)-\delta)$ pairs of this type contributing to the dimension.

Adding these two parts, the dimension of the filling is at least $\sum_{\delta=1}^{n}(h(\delta)-\delta)+$ $\sum_{i=1}^{\ell} \sum_{j=2}^{k_{i}}(j-1) d_{i, j}$. Since this is also the upper bound for the dimension, the dimension must equal to this sum.

\section{The Dimension when $h(\alpha)=\alpha+1$}

We will first prove a sharper upper bound for the dimension of a filling when $h(\alpha)=\alpha+1$ (and $h(n)=n$ ), and then show that this upper bound is achieved in a particular filling. 
Recall that $d_{i, j}$ is the length of the $j^{\text {th }}$ row of the $i^{\text {th }}$ tableau (both counted from the top downward), and $k_{i}$ is the number of rows in the $i^{\text {th }}$ tableau. Let $k_{\max }$ be the maximum of all the $k_{i}$, meaning $k_{\max }$ is the largest number of rows in any tableau of the given multitableau.

Lemma 6.1. Let $\lambda$ be a multitableau and $\lambda^{\prime}$ be a multitableau obtained from $\lambda$ by reordering the tableaux. The maximal $h$-dimension of $\lambda$ is the same as the maximal $h$-dimension of $\lambda^{\prime}$.

Proof. Reordering the multitableaux is equivalent to conjugating the corresponding linear operator $X$ by the permutation $w$. By [T, Proposition 2.7], the Hessenberg variety $H e(X, h)$ is homeomorphic to $H e\left(w^{-1} X w, h\right)$. In particular, they have the same dimension. From [T, Theorem 7.1], the dimension of a Hessenberg variety is the maximal $h$-dimension of its corresponding multitableau. (The proof of [T, Theorem 7.1] does not depend on the order of the generalized eigenspaces in $X$.)

Lemma 6.2. For each entry $\alpha$ and each row in the same tableau as $\alpha$, excluding the one containing $\alpha$, there are at most 2 entries which are $D_{\alpha}$ in that row. Moreover, if there are $2 D_{\alpha}$ entries in a row, then $\alpha-1$ is also in this row and is immediately to the right of the left $D_{\alpha}$ entry.

Proof. Suppose there are at least three entries $\beta, \gamma$, and $\delta$ which are all $D_{\alpha}$ in some row not containing $\alpha$. Without loss of generality, assume the entries are arranged as follows (possibly with $\epsilon$ and $\gamma$ or $\zeta$ and $\delta$ coinciding).

\begin{tabular}{|l|l|l|l|l|l|l|}
\hline$\beta$ & $\epsilon$ & $\cdots$ & $\gamma$ & $\zeta$ & $\cdots$ & $\delta$ \\
\hline
\end{tabular}

Note that if $\beta_{1}>\beta_{k}$, then the subtableau \begin{tabular}{|l|l|l|}
\hline$\beta_{1}$ & $\cdots$ & $\beta_{k}$ \\
\hline
\end{tabular} is allowed if and only if $\beta_{i}=$ $\beta_{1}-(i-1)$ for $1 \leq i \leq k$. This can be proved by induction on $k$ : suppose $\beta_{i}=\beta_{1}-(i-1)$ for all $1 \leq i<k$. If $\beta_{k}<\beta_{k-1}$, then by definition of allowed fillings, $\beta_{k}=\beta_{1}-(k-1)$. Otherwise, $\beta_{k}>\beta_{1}$ since all numbers in the interval $\left[\beta_{1}, \beta_{k-1}\right]$ already appear in the tableau. Thus, as $\alpha>\gamma$, if $\epsilon>\alpha$, then $\alpha$ would appear in this row, contrary to our assumption. Hence, $\alpha>\epsilon$. Also $\alpha \leq \epsilon+1$ since $\beta$ is $D_{\alpha}$, so $\alpha=\epsilon+1$. By the same argument, $\alpha=\zeta+1$, a contradiction, as $\epsilon \neq \zeta$.

Also, if there are two $D_{\alpha}$ entries in a row, then by our argument above, if $\beta$ and $\gamma$ were the two $D_{\alpha}$ entries, then $\epsilon=\alpha-1$.

Theorem 6.3. For a multitableau $\lambda$ with the $j^{\text {th }}$ row of the $i^{\text {th }}$ tableau having length $d_{i, j}$, and the Hessenberg function $h(\alpha)=\alpha+1$, if $k_{\max }$ is the largest number of rows in any tableau, then the dimension of any filling is at most

$$
n-k_{\max }+\sum_{i=1}^{\ell} \sum_{j=2}^{k_{i}}(j-1) d_{i, j} .
$$


Proof. By Lemma 6.1 we may assume that the bottom tableau has the largest number of rows, so $k_{\ell}=k_{\max }$, where there are $\ell$ tableaux in $\lambda$. We will use the expression for the dimension from Corollary 2.9 to give the upper bound.

Recall from Remark 2.7 that whenever $\beta$ is $D_{\alpha}$ and in the same row as and to the right of $\alpha$, then $\beta$ has a contributing $P_{\alpha}$ entry.

Breaking up the first part of the sum in Corollary 2.9 into pairs where both $\alpha$ and $\beta$ are in the same row, and pairs where they are in different rows, and applying Lemma 6.2, we can rephrase this first part as the sum of:

(a) the number of pairs $(\alpha, \beta)$ where $\alpha$ and $\beta$ are in the same row, $\beta$ is to the right of $\alpha$ and $\beta$ is $P_{\alpha}$,

(b) the sum over all $\alpha$ of the number of rows (excluding the row containing $\alpha$ ) which have two entries $\beta$ and $\gamma$ which are both to the right of or directly above $\alpha$, and such that $\beta$ and $\gamma$ are both $D_{\alpha}$, and

(c) the sum over all $\alpha$ of the number of rows (excluding the row containing $\alpha$ ) which have at least one entry $\beta$ which is to the right of or directly above $\alpha$, and such that $\beta$ is $D_{\alpha}$.

First note that for this particular Hessenberg function $\beta$ is $P_{\alpha}$ if and only if $\beta=\alpha-1$. So (a) is simply equal to the number of entries $\beta$ for which $\beta+1$ is to the left of $\beta$ in the same row. Also, (b) is, by Lemma 6.2, at most the number of entries $\epsilon$ for which $\epsilon+1$ is to the left of $\epsilon$ and in the same tableau as $\epsilon$, but in a different row from $\epsilon$.

We now give an upper bound for (c). Given any two entries $\alpha_{1}$ and $\beta_{1}$ which are in the same column such that $\beta_{1}$ is above $\alpha_{1}$, we show that there can be an entry $\beta_{k}$ in the same row as $\beta_{1}$ which is $D_{\alpha_{1}}$ contributing to the dimension, or an entry $\alpha_{\ell}$ in the same row as $\alpha_{1}$ which is $D_{\beta_{1}}$ contributing to the dimension, but not both. Using the notation in Figure 6, if $\beta_{1}>\alpha_{1}$, suppose that $\beta_{k}$ is $D_{\alpha_{1}}$. We then have $\beta_{1}>\alpha_{1}>\beta_{k}$, but $\alpha_{1}$ is in a different row than $\beta_{1}$ and $\beta_{k}$, so by the principle used in the proof of Lemma 6.2, this is not possible. If $\alpha_{1}>\beta_{1}$, then suppose that $\alpha_{\ell}$ is $D_{\beta_{1}}$. We then have $\alpha_{1}>\beta_{1}>\alpha_{\ell}$, and again by the same reason, this is not possible. Therefore, (c) is at most $\sum_{c \in C_{i}}\left(\begin{array}{c}\ell(c) \\ 2\end{array}\right)=\sum_{i=1}^{\ell} \sum_{j=2}^{k_{i}}(j-1) d_{i, j}$.

So far, we have shown that the first part of the sum in Corollary 2.9 is at most the sum of $\sum_{i=1}^{\ell} \sum_{j=2}^{k_{i}}(j-1) d_{i, j}$, the number of entries $\beta$ for which $\beta+1$ is to the left of $\beta$ and in the same row, and the number of entries $\beta$ for which $\beta+1$ is to the left of $\beta$ and in a different row of the same tableau. Note that the second part of the sum in Corollary 2.9 is exactly the sum over all entries $\beta$ for which $\beta+1$ is in a different tableau below the one containing $\beta$. Thus, in order to get an upper bound for the dimension, we add $\sum_{i=1}^{\ell} \sum_{j=2}^{k_{i}}(j-1) d_{i, j}$ to the sum of

- the number of entries $\beta$ where $\beta+1$ is to the left of $\beta$ and in the same row,

- the number of entries $\beta$ where $\beta+1$ is in a different tableau below the one containing $\beta$, and 
- the number of entries $\beta$ where $\beta+1$ is in the same tableau but in a different row from $\beta$, and $\beta+1$ is to the left of $\beta$.

For any entry $\beta$, at most one of the above three situations can occur. Also, for any entry $\beta$ which is not in the left column of the bottom tableau, any one of the three could occur. If $\beta$ is in the left column of the bottom tableau, there can be nothing to the left of $\beta$ and nothing in a tableau below the one containing $\beta$. Therefore we can have at most one of these situations occurring for each entry $\beta$ which is not in the left column of the bottom tableau. There are $n-k_{\ell}$ such possible entries $\beta$, as desired.

For a multitableau satisfying the condition that $k_{\ell} \geq k_{i}$ for all $i$, and the Hessenberg function $h(\alpha)=\alpha+1$, consider the following filling of $\lambda$ : first put the first $d_{1,1}$ numbers in the top row of the top tableau, in decreasing order, then put the next $d_{2,1}$ numbers in the top row of the next tableau, again in decreasing order. Continue this process, putting the numbers in decreasing order in the top row of each tableau, continuing downward to each tableau. After this is done, go back to the top tableau and put the next $d_{1,2}$ numbers in the second row of the top tableau, in decreasing order. Continue this same process going down the tableaux for the second row of each. Then continue for the third rows, fourth rows, and so on, skipping a tableau if all of its rows have been filled. Since the bottom tableau has the greatest number of rows, it will be filled last. We call this filling the large $\alpha+1$ filling. An example of this filling is given in Figure 8 .

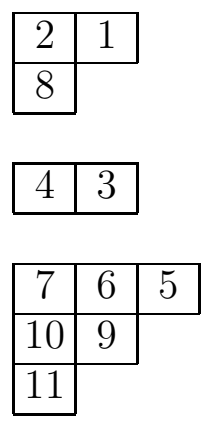

Figure 8: An example of the large $\alpha+1$ filling.

Proposition 6.4. When the bottom tableau has the largest number of rows, (meaning $\left.k_{\ell}=k_{\max }\right)$, the dimension of the large $\alpha+1$ filling is

$$
n-k_{\ell}+\sum_{i=1}^{\ell} \sum_{j=2}^{k_{i}}(j-1) d_{i, j}
$$

Proof. If $\beta$ is $P_{\alpha}$, this means that $\beta<\alpha \leq h(\beta)=\beta+1$. So if $\beta$ is $P_{\alpha}$, this means that $\alpha=\beta+1$. Using this fact to slightly modify the expression of the dimension from Corollary 2.10, the dimension of a filling is the sum of 
1. the number of $\beta<n$ in the filling of $\lambda$ such that

- $\beta+1$ is in the same tableau as $\beta$ and

- the box filled with $\beta+1$ is to the left of or directly below the box filled with $\beta$.

2. the number of pairs $(\alpha, \beta)$ in the filling of $\lambda$ such that

- $\alpha$ and $\beta$ are in the same tableau,

- the box filled with $\alpha$ is to the left of or directly below the box filled with $\beta$,

- $\beta$ is $D_{\alpha}$ with no contributing $P_{\alpha}$, meaning $\alpha-1$ is not anywhere directly to the left of $\beta$ and above $\alpha$.

3. the number of $\beta<n$ in $\lambda$ such that

- $\beta+1$ and $\beta$ are in different tableaux and

- the tableau containing $\beta+1$ is below the tableau containing $\beta$.

Consider part (1) of this sum. By construction of the filling, if the entry $\beta$ is not at the beginning of a row, then $\beta \neq n$ and $\beta+1$ is to the left of and in the same tableau as $\beta$. The number of such boxes is $n-\left(k_{1}+k_{2}+\cdots+k_{\ell}\right)$. If $\beta$ is at the beginning of a row, then $\beta+1$ will be in a tableau below the one containing $\beta$, and so does not add to part (1) of the dimension. Thus part (1) contributes $n-\left(k_{1}+k_{2}+\cdots+k_{\ell}\right)$ to the dimension.

Similarly, for part (3) of the sum, the number of entries $\beta$ with $\beta \neq n$ such that $\beta+1$ is in a tableau below the one containing $\beta$ is equal to the number of boxes at the beginning of a row not in the bottom tableau, which is $k_{1}+k_{2}+\cdots+k_{\ell-1}$.

If $\alpha$ and $\beta$ are in different rows with $\beta$ not at the end of a row, then $\beta-1$ is immediately to the right of $\beta$. If $\alpha>\beta$, then we also have $\alpha>h(\beta-1)=\beta$. Thus $\beta$ cannot be $D_{\alpha}$. So, the number of pairs $(\alpha, \beta)$ satisfying the conditions in part (2) of the sum is equal to the number of pairs $(\alpha, \beta)$ where $\beta$ is at the end of a row which does not contain $\alpha$, the entry $\alpha$ is to the left of or directly below $\beta$, and $\beta$ is $D_{\alpha}$, meaning $\alpha>\beta$.

So for part (2) of the sum, we need to count the number of pairs $(\alpha, \beta)$ such that $\alpha$ and $\beta$ are in the same tableau, $\beta$ is at the end of a row, $\alpha$ is to the left of or directly below $\beta$, and $\alpha>\beta$. For each entry $\delta$, every entry $\gamma$ below $\delta$ has $\delta<\gamma$ by the rules of our filling. But any entry $\gamma$ above $\delta$ in the same tableau has $\delta>\gamma$, and if $\gamma$ is at the end of a row above $\delta$, then $\delta$ is to the left of or directly below $\gamma$. Thus the number of this type of pair is equal to $\sum_{\alpha} r(\alpha)=\sum_{i=1}^{\ell} \sum_{j=2}^{k_{i}}(j-1) d_{i, j}$, where $r(\alpha)$ is the number of rows above $\alpha$ in the same tableau.

Adding up these three parts, we get the desired dimension for this filling.

Corollary 6.5. For a multitableau $\lambda$ with the $j^{\text {th }}$ row of the $i^{\text {th }}$ tableau having length $d_{i, j}$, and $k_{i}$ rows in the $i^{\text {th }}$ tableau, such that $k_{\ell}>k_{i}$ for all $i$, if the Hessenberg function is $h(\alpha)=\alpha+1$, then the maximal dimension of allowed fillings is exactly

$$
n-k_{\max }+\sum_{i=1}^{\ell} \sum_{j=2}^{k_{i}}(j-1) d_{i, j} .
$$


Proof. For any multitableau $\lambda$, by Lemma 6.1, the maximal dimension of allowed fillings is the same as the maximal dimension for the multitableau $\lambda^{\prime}$, where $\lambda^{\prime}$ is obtained from $\lambda$ by reordering the tableau so that the bottom one contains the largest number of rows.

\section{The Dimension of the Big Filling}

We now consider the big filling, which corresponds to the intersection of the big cell in the flag variety with a Hessenberg variety. In this filling, we put $n$ in the bottom left entry of the bottom tableau, put $n-1$ above it, and continue up the column with numbers decreasing. When a column is full, we begin filling the column to the right of it from the bottom to the top with decreasing numbers, moving up to the bottom left entry of the next tableau if one is full. For example, see Figure 9.

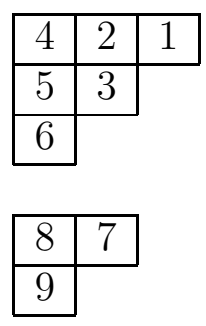

Figure 9: An example of the big filling.

Let $s_{j}$ denote the number of entries in the $j^{\text {th }}$ tableau (counted from the top tableau downward). Then $\sum_{i=1}^{m} s_{i}$ is the largest entry in the $m^{\text {th }}$ tableau of the big filling.

Proposition 7.1. When the big filling is allowed, its dimension is

$$
\sum_{\alpha=1}^{n}(h(\alpha)-\alpha)+\sum_{m=1}^{\ell} \sum_{\delta \in L_{m}} \max \left\{\left(\sum_{i=1}^{m} s_{i}\right)-h(\delta), 0\right\},
$$

where $L_{m}$ is the set of entries in the left column of the $m^{\text {th }}$ tableau and $\ell$ is the number of tableaux in $\lambda$.

Proof. We use the expression for the dimension given in Corollary 2.10. For each $\alpha$, any $\beta$ such that $\beta<\alpha \leq h(\beta)$ is, by the definition of the big filling, either in a tableau above the one containing $\alpha$, or else $\alpha$ and $\beta$ are in the same tableau with the box filled with $\beta$ directly above or to the right of the one filled with $\alpha$.

Thus, the total number of $P_{\alpha}$ entries which are contributing to the dimension is $\sum_{\beta=1}^{n}(h(\beta)-\beta)$, so the dimension is equal to the sum of the two quantities:

1. $\sum_{\beta=i}^{n}(h(\beta)-\beta)$ and

2. the number of pairs $(\alpha, \beta)$ where $\alpha$ is to the left of or directly below $\beta$ and $\beta$ is $D_{\alpha}$ with no contributing $P_{\alpha}$. 
Suppose $\alpha$ is not in the left column and $\beta_{m}$ is any entry in one of the three relative positions $\beta_{1}, \beta_{2}$, or $\beta_{3}$ seen in Figure 10 (meaning $\beta_{m}$ is either directly above $\alpha$, immediately to the right of $\alpha$, or below $\alpha$ and in the column immediately to the right of the one containing $\alpha$ ). Since $\alpha$ is to the left of or directly below $\beta_{m}$, we have $\alpha>\beta_{m}$. Also, in the middle case, $\alpha \leq h\left(\beta_{2}\right)$, since the filling is allowed, and when $m=1$ or 3 , then $\gamma_{m}>\alpha$ by the rules for this filling. Also, $\gamma_{m} \leq h\left(\beta_{m}\right)$, so it follows that $\beta_{m}<\alpha \leq h\left(\beta_{m}\right)$ in any of the three cases. Thus $\beta_{m}$ is $P_{\alpha}$. By the same argument, if $\alpha$ is in the left column, $\beta_{2}$ and $\beta_{3}$ will be $P_{\alpha}$.

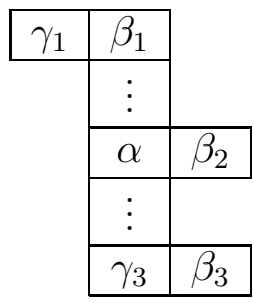

Figure 10: The three relative positions for $\beta_{m}$.

Recall that if an entry $\beta$ is both $D_{\alpha}$ and $P_{\alpha}$, we say that it has a contributing $P_{\alpha}$. Thus, the only possible way to have an entry $\beta$ be $D_{\alpha}$ with no contributing $P_{\alpha}$ is if $\alpha$ is in the left column and $\beta$ is in a row above the one containing $\alpha$. Hence the number of pairs $(\alpha, \beta)$ where $\alpha$ is to the left of or directly below $\beta$ and $\beta$ is $D_{\alpha}$ with no contributing $P_{\alpha}$ is equal, by the Slide Left Lemma, to the number of pairs $(\alpha, \beta)$ where $\alpha$ is in the left column and $\beta$ is the furthest right entry in a row above $\alpha$. Given any entry $\beta$ that is the right entry in a row above $\alpha$ and in the same tableau, $\alpha>\beta$ in this filling, so $\beta$ is $D_{\alpha}$. We only need to consider for all $\alpha$ in the left column of each tableau, the rows above $\alpha$ in the same tableau for which the furthest left entry is not $P_{\alpha}$, as if it does not happen there, it will not happen further to the right. We can rephrase this quantity as the sum over all entries $\gamma$ in the left column of the number of entries $\alpha$ below $\gamma$ in the same column and same tableau for which $\gamma$ is not $P_{\alpha}$.

If $s_{j}$ is the total number of boxes in the $j^{\text {th }}$ tableau, the largest entry in the $m^{\text {th }}$ tableau (counted from the top) is $\sum_{j=1}^{m} s_{j}$. Any $\gamma$ in the $m^{\text {th }}$ tableau is $P_{\alpha}$ for $\alpha=$ $\gamma+1, \gamma+2, \ldots, h(\gamma)$. In order to count the number of entries $\alpha$ below a given $\gamma$ in the same column and same tableau for which $\gamma$ is not $P_{\alpha}$, we consider two cases:

1. If $h(\gamma) \geq \sum_{j=1}^{m} s_{j}$, then for every entry $\alpha$ in the same tableau as $\gamma$ and below $\gamma$, we see that $\gamma$ is $P_{\alpha}$. In this case, the number of entries $\alpha$ below $\gamma$ in the same column and same tableau for which $\gamma$ is not $P_{\alpha}$ is zero, and we see that $\max \left\{\left(\sum_{j=1}^{m} s_{j}\right)-\right.$ $h(\gamma), 0\}=0$.

2. If $h(\gamma)<\sum_{j=1}^{m} s_{j}$, then we know that $\gamma$ is not $P_{\alpha}$ for $\alpha=h(\gamma)+1, h(\gamma)+$ $2, \ldots, \sum_{j=1}^{m} s_{j}$, of which there are $\sum_{j=1}^{m} s_{j}-h(\gamma)$ possibilities. Each of these occurs below $\gamma$, so the number of entries $\alpha$ below $\gamma$ in the same column and same tableau 
for which $\gamma$ is not $P_{\alpha}$ is $\sum_{j=1}^{m} s_{j}-h(\gamma)$, and in this case, $\max \left\{\left(\sum_{j=1}^{m} s_{j}\right)-h(\gamma), 0\right\}=$ $\left(\sum_{j=1}^{m} s_{j}\right)-h(\gamma)$.

Thus there are $\sum_{\delta \in L_{m}} \max \left\{\left(\sum_{i=1}^{m} s_{i}\right)-h(\delta), 0\right\}$ pairs of this type in the $m^{\text {th }}$ tableau, where $L_{m}$ is the set of entries in the left column of the $m^{\text {th }}$ tableau. Summing this up over all $m$ and adding to $\sum_{\alpha=1}^{n}(h(\alpha)-\alpha)$, gives the dimension of the filling.

Example 7.2. When we have the Hessenberg function

\begin{tabular}{c||c|c|c|c|c|c|c|c|c}
$\alpha$ & 1 & 2 & 3 & 4 & 5 & 6 & 7 & 8 & 9 \\
\hline$h(\alpha)$ & 3 & 5 & 5 & 5 & 5 & 7 & 8 & 9 & 9
\end{tabular}

the dimension of the filling seen in Figure 9, is

$$
\sum_{\alpha=1}^{9}(h(\alpha)-\alpha)+\sum_{\gamma \in\{4,5,6\}} \max \{6-h(\gamma), 0\}+\sum_{\gamma \in\{8,9\}} \max \{9-h(\gamma), 0\}=13
$$

Remark 7.3. Proposition 7.1 gives a lower bound on the maximum dimension of a filling when the big filling is allowed. Thus the maximum dimension is bounded below by (1). This also gives a lower bound for the dimension of the corresponding Hessenberg variety.

Conjecture 7.4. We conjecture that whenever the big filling is an allowed filling, it gives the maximum dimension for any filling, and is thus the dimension of the corresponding Hessenberg variety.

\section{References}

[DPS] F. de Mari, C. Procesi, and M. A. Shayman, Hessenberg varieties, Trans. Amer. Math. Soc. 332 1992, 529-534.

[DS] F. de Mari and M. A. Shayman, Generalized Eulerian numbers and the topology of the Hessenberg variety of a matrix, Acta Appl. Math. 12 1988, 213-235.

[F] J. Fulman, Descent identities, Hessenberg varities, and the Weil conjectures, J. Combin. Theory Ser. A 87 1999, 390-397.

[S] R. P. Stanley, Enumerative Combinatorics, Vol. 1, Cambridge Studies in Advanced Mathematics 49, Cambridge University Press, Cambridge, 1997.

[ST] E. Sommers and J. Tymoczko, Exponents for B-stable ideals, to appear in the Transactions of the American Mathematical Society. Available at arXiv:math.AG/0406047.

[T] J. Tymoczko, Linear conditions imposed on flag varieties, to appear in the American Journal of Mathematics. Available at arXiv:math.AG/0406541. 